\title{
Factores de riesgo asociados a la deserción estudiantil universitaria en programas de pregrado de la Universidad de San Buenaventura, Bogotá (2009-2013) ${ }^{1}$
}

\author{
Floralba Barrero Rivera ${ }^{2}$, Claudia Barrero Espinosa ${ }^{3}$, Harold Borja Cely ${ }^{4} \&$ Merfi Raquel Montaño Sinisterra ${ }^{5}$ \\ Universidad de San Buenaventura, Colombia \\ Recibido, mayo 14 de 2015 \\ Concepto evaluación, septiembre 8 de 2015 \\ Aceptado, octubre 15 de 2015 \\ Referencia: Barrero Rivera, F.; Barrero Espinosa, C.; Borja Cely, \\ H.; Montaño Sinisterra M. (2015). "Factores de riesgo asociados \\ a la deserción estudiantil universitaria en programas de pregrado \\ de la Universidad de San Buenaventura, Bogotá (2009-2013)”. \\ Revista Academia y Virtualidad, 8, (2), 60-72
}

\section{Resumen}

Esta investigación presenta una tendencia general, mas no definitiva, de los factores de riesgo asociados a la deserción estudiantil universitaria en programas de pregrado de la Universidad de San Buenaventura, Bogotá (2009-2013). Se desarrolló una investigación mixta de carácter descriptivo. Así, se construyó y validó un instrumento con el propósito de conocer los factores de riesgo (individual, institucional, socioeconómico, académico).

Los resultados mostraron que los factores de riesgo asociados a la deserción universitaria en este contexto educativo corresponden al factor académico, seguido por el institucional, individual y socioeconómico. El estudio propone desde la psicología educativa intervenir en estos aspectos con modelos asociados a cada uno de ellos, donde se logre sistematizar el comportamiento de la deserción y la intervención de la misma con fines de prevenir y mitigar el fenómeno.

Palabras clave: educación, factores de riesgo, deserción estudiantil.

1. Este artículo se configura como parte de los resultados de la investigación "Factores de riesgo asociados a la deserción estudiantil universitaria en Programas de Pregrado de la Universidad de San Buenaventura, Bogotá (2009-2013) “, desarrollada por investigadores del Grupo Tendencias Actuales en Educación y Pedagogía (TAEPE) durante 2014 en la Universidad de San Buenaventura, Bogotá.

2. Magister En Desarrollo Educativo y Social. Profesora de la Universidad de San Buenaventura. Correo: fbarrero@usbbog.edu.co

3. Directora especializaciones Pedagogía y Docencia Universitaria Didáctica para lecturas y escrituras con énfasis en literatura. Universidad San Buenaventura.Correo: cbarrero@usbbog.edu.co

4. Director de la Unidad de Bienestar Institucional. Universidad San Buenaventura. Correo: hborja@usbbog.edu.co

5. Psicóloga, Unidad de Bienestar Institucional. Universidad San Buenaventura. Correo: mrmontaño@usbbog.edu.co 


\section{Risk factors related to undergraduate student dropout rate at Universidad de San Buenaventura, Bogotá (2009-2013)}

\section{Abstract}

This research shows an overview about several dimensions to get some risk factors related to student dropout rate in academic programs at Universidad de San Buenaventura during period 2009-2013. A mixed descriptive research was developed to create and validate an instrument in order to know such risks. Results showed the academic issue as a main factor, following the institutional, individual and socioeconomic events. Our research proposes from the educational psychology to intervene all those factors by taking models, which involve the entire factors and also systematizing the behavior of the student dropout rate in order to prevent and moderate this issue.

Keywords: education, risk factors, student dropout rate.

\section{Fatores de risco associados com a deserção estudantil universitária em programas de pre-graduação na Universidade de San Buenaventura, Bogotá (2009-2013)}

\section{Resumo}

Esta pesquisa apresenta uma tendência geral, embora não definitiva, dos fatores de risco associados com a deserção estudantil universitária em programas de pre-graduação na Universidade de San Buenaventura, Bogotá (2009.2013). Desenvolveu-se uma pesquisa mista de caráter descritivo. Assim, construiu-se e validou-se um instrumento com o propósito de conhecer os fatores de risco (individual, institucional, socioeconômico, acadêmico).

Os resultados mostraram que os fatores de risco associados com a deserção universitária neste contexto educativo correspondem ao fator acadêmico, seguido pelo institucional, individual, e socioeconômico. $\mathrm{O}$ estudo propõe desde a psicologia educativa, intervir nesses aspetos com modelos associados a cada um deles, onde se consiga sistematizar o comportamento da deserção e a intervenção da mesma com fins de prevenir e mitigar o fenômeno.

Palavras chave: educação, fatores de risco, deserção estudantil.

\section{Introducción}

Educación para todos es un propósito de la Unesco, en sus distintos documentos. En ese marco referencial el principal punto de reflexión tiene que ver con la deserción estudiantil entendida como uno de los problemas que aborda la mayoría de las instituciones de Educación Superior tanto de América Latina como del mundo. La misión de la Educación Superior es un punto que convoca a la reflexión según se puede afirmar que sin educación no hay desarrollo. Desde esta aseveración, la innovación y los avances significativos en ciencia y tecnología se ven seriamente afectados.

En Colombia los diversos estudios que se han llevado a cabo sobre este fenómeno condujeron al Ministerio de Educación Nacional a concluir que: 
1)Eldesarrollodeinvestigacionesdesdelaperspectiva sectorial era insuficiente, constituyéndose en una limitante para la elaboración de políticas públicas

(2) Particularmente, el conocimiento de las diferentes causas de abandono en Educación Superior se convierte en un insumo fundamental para mejorar los procesos y resultados de la formulación e implementación de políticas, estrategias y acciones, tanto al nivel de las instituciones de Educación Superior, como en el ámbito nacional

(3) El fenómeno de la deserción compromete la estabilidad de los ingresos de las instituciones de Educación Superior y la calidad de sus procesos administrativos y académicos. Así mismo, afecta directamente las metas de cobertura y accesibilidad y se constituye en un costo social que es asumido por las familias, las instituciones de Educación Superior y el Estado (MEN, 2004: 1).

Las estadísticas entonces muestran que en Colombia el índice de deserción para los años 90, osciló entre 68\% y $47 \%$ de los estudiantes matriculados (Icfes, 2003). Según datos suministrados por el Ministerio de Educación Nacional, la tasa anual de deserción estudiantil de pregrado fue $13 \%$ para el primer semestre del 2005 , pero si se mide por cohortes, es decir, si se toma el número de estudiantes que se gradúan del total que ingresan en un mismo periodo, $52 \%$ de los estudiantes colombianos que empiezan una carrera universitaria, no la concluyen.

Es decir, que de cada dos estudiantes que se matriculan en un programa de pregrado, solo uno culmina sus estudios. Aunque se ha presentado una tendencia al descenso con porcentajes del $51 \%$ para $2003 ; 50.2 \%$ para $2004 ; 48.4 \%$ para 2005; y $47.5 \%$ para 2006 , las cifras aún son altas (informe Ministerio de Educación Nacional, 2007).

Desde lo enunciado todos los agentes educativos de la instituciones de Educación Superior están llamados a comprometerse con el tema, en este sentido esta investigación pretende contribuir con la historia investigativa del fenómeno, como también aportar un instrumento validado que permita de manera contextual realizar un seguimiento a la deserción estudiantil universitaria.

En la Universidad de San Buenaventura, sede Bogotá, no hay investigaciones actualizadas que expliquen los orígenes de los factores asociados a esta temática. Por tanto, es prioritario conocer los factores de riesgo los cuales se definen como

[..] cualquier característica o circunstancia detectable de una persona o grupo de personas que se sabe asociada con un aumento en la probabilidad de padecer, desarrollar o estar especialmente expuesto a un proceso mórbido.

Estos factores de riesgo (biológicos, ambientales, de comportamiento, socioculturales, económicos) pueden, sumándose unos a otros, aumentar el efecto aislado de cada uno de ellos produciendo un fenómeno de interacción (Redondo, 1999).

Así, se puede afirmar que los estudiantes que llegan a la Universidad con bajo capital cultural y social son los más opcionados para desertar. (Tinto, 1975; García (2005); Vahos (2002) citados por Ministerio de Educación Nacional (2007).

En este marco de reflexión, la deserción estudiantil se entiende como el abandono a la vida académica. Gómez (1998) define la deserción como un "fracaso personal temprano cuya marca dura para toda la vida" (p.54).

Tinto (1993) define la deserción como una situación a la que se enfrenta un estudiante cuando aspira y no logra concluir su proyecto educativo. Luego, es posible considerar como desertor a aquel individuo que siendo estudiante de una institución de Educación Superior no presenta actividad académica durante tres semestres consecutivos. En esta perspectiva, Tinto considera que existen varios períodos críticos en la trayectoria estudiantil en que las interacciones entre la institución y los estudiantes pueden influir directamente en la deserción. 
El primer momento se desarrolla en el proceso de admisión cuando el estudiante realiza el primer contacto con la universidad. Durante la etapa de indagación de los requisitos para ingresar a una determinada institución, los estudiantes forman las primeras impresiones. Una segunda etapa crítica en la trayectoria académica del estudiante tiene que ver con la transición entre la enseñanza media y la institución. Esto sucede porque al estudiante le cuesta adaptarse a la vida universitaria.

En esta investigación se asume el concepto de Tinto acerca de la deserción estudiantil, concebida como el estudiante que aspira y no logra concluir su proyecto educativo. El grupo investigador consideró como criterio de deserción la inactividad académica de dos semestres consecutivos, tal como lo plantea el Ministerio de Educación Nacional.

(2007). Por otra parte, la investigación sustenta diferentes modelos conceptuales para explicar la deserción estudiantil. Se encuentran los modelos psicológicos, los cuales explican la deserción desde los rasgos de personalidad donde se diferencian a los estudiantes que terminan los estudios con aquellos que no lo logran.

Fishbein y Ajzen (1975) proponen la teoría de la acción razonada que analiza el comportamiento como actitudes de respuesta a objetivos específicos, considerando normas subjetivas que guían el comportamiento hacia esos objetos y el control percibido por ese comportamiento. Este modelo se asocia a los factores de riesgo individual que según se identifican en aspectos personales, entre éstos salud y condiciones emocionales de los estudiantes, entre otros.

Por otra parte, el rendimiento académico entendido como factor de riesgo, se considera y explica desde el modelo de elección académica de Ethington (1990), el cual se basa en diferentes marcos teóricos-empíricos (toma de decisiones). Ethington introduce una teoría más general acerca de las conductas de logro, y con ello concluye que el rendimiento académico previo afecta el desempeño futuro al actuar sobre el autoconcepto, su percepción de las dificultades del estudio, sus metas, valores y expectativas de éxito.
Este modelo explica el factor de riesgo académico, el cual se refleja en las distintas percepciones de los estudiantes con respecto a aspectos como orientación vocacional, historia académica, evaluación, interacción con los profesores, reglamento estudiantil, etc.

También se encuentra el modelo económico, pues según las investigaciones desarrolladas por Cabrera (1992, 1993) se distinguen modelos costo-beneficio, que consisten en identificar las ventajas de los beneficios sociales y económicos asociados a los estudiantes, los cuales son percibidos como mayores que los derivados por actividades alternas, como por ejemplo un trabajo. Los beneficios son percibidos por los estudiantes como muchos mayores y por esa razón no desertan.

Desde esta perspectiva, este modelo plantea intervenir este aspecto socioeconómico con la entrega de subsidios que constituyen una forma definitiva para contrarrestar la deserción estudiantil. Este modelo busca privilegiar el impacto definitivo de los beneficios estudiantiles sobre la deserción estudiantil. Se pueden reflejar los factores de riesgo socioeconómicos los cuales están conformados por las dificultades económicas, la caracterización sociodemográfica de las familias, entre otros.

Finalmente, encontramos el modelo organizacional, que sostiene que la deserción estudiantil universitaria depende de las cualidades de la organización en la integración social, y más particularmente en el abandono de los estudiantes que ingresan a ella (Berger y Milem 2000). En este enfoque es altamente relevante la calidad de la institución, su misión, visión y demás aspectos que hacen de la institución un lugar muy atractivo para permanecer en ella.

En este modelo se pueden reflejar los factores institucionales, tales como proyecto educativo, políticas institucionales de permanencia de estudiantes, entre otros. Como puede entenderse, cada factor de riesgo de la deserción estudiantil está ampliamente explicado y sustentado teórica y empíricamente por cada uno de los modelos mencionados. La presente investigación considera de gran valor la intervención de la psicología 
educativa, para realizar un puente entre la explicación del factor de riesgo tanto individual, socioeconómico, académico e institucional, convirtiendo esta comprensión del factor en una oportunidad de intervención y mejoramiento.

Desde la psicología educativa se pueden formular programas que puedan ser sistemáticamente validados con los aportes teóricos de cada modelo aplicando programas educativos inspirados en el sustento teórico de cada uno de ellos, e interviniendo desde programas específicos.

Esta investigación pretende contribuir una vez más a ofrecer elementos de análisis que permitan enriquecer la comprensión de esta problemática. A partir del conocimiento de los factores de riesgo tanto de orden individual, socioeconómico, institucional y académico, se pueden ofrecer análisis que contribuyan a mitigar los mismos y una vez analizados a profundidad puedan orientar las intervenciones desde los modelos asociados a cada uno de ellos iluminando así los programas de intervención.

\section{Método}

En este ejercicio se realizó una investigación descriptiva en la cual se especificaron los factores de riesgos asociados a la deserción (Hernández, 2010). El enfoque metodológico fue mixto por cuanto se aprovecharon en la misma los datos minuciosamente encontrados, lo cual permite una visión profunda del fenómeno objeto de estudio (Hernández Sampieri, 2010; Sandoval, 2002).

\section{Participantes}

Para este trabajo se realizó un muestreo no probabilístico y voluntario; la elección de los participantes no dependió de la probabilidad, sino de causas relacionadas con las características del equipo investigador, quien diseñó la muestra (Sandoval, 2002:136) (Hernández, 2010: 176). La muestra estuvo conformada por 77 estudiantes con una edad promedio de 25 años, quienes de manera voluntaria accedieron a diligenciar el instrumento vía web, 61\% fueron hombres y $36 \%$ mujeres.
Para los grupos focales se contó con la participación de cinco facultades (Ingeniería, Educación, Psicología, Ciencias Empresariales, Ciencias Jurídicas y Políticas). Los programas que participaron en el grupo focal fueron: Ingeniería de Sonido, Ingeniería Mecatrónica, Ingeniería Electrónica, Ingeniería de Telecomunicaciones, Ingeniería de Sistemas, Ingeniería Aeronáutica, Administración de Empresas, Contaduría, Economía, Licenciatura en Educación para la Primera Infancia, Licenciatura en Educación Básica con Énfasis en Lengua Inglesa, Psicología, Derecho, Relaciones Internacionales y Ciencia Política.

\section{Instrumentos}

Para la recolección de la información se utilizaron dos instrumentos (una encuesta y sesiones de grupos focales). La encuesta fue diseñada y validada por el equipo investigador, en la que se siguieron todos los aspectos metodológicos que exige la psicometría en la construcción y validación de un instrumento: 1) diseño y elaboración de la encuesta; 2) validación de contenido con jueces expertos, especializados, Internos y externos; 3) validación de constructo (Alfa de Cronbach); 4) pilotaje del instrumento y 5) versión instrumento final.

Los grupos focales se desarrollaron con directores de programas y profesores consejeros, el objetivo fundamental fue crear o recrear escenarios o situaciones que propiciaran la recolección de la información, escuchando la percepción de los participantes acerca del tema objeto de estudio, en este caso la deserción estudiantil. (Sandoval, 2002:136). Se fraccionó la información en unidades temáticas por medio de párrafos. Desde la técnica de análisis de contenido, la cual realiza inferencias válidas y confiables de datos para analizar la comunicación en un contexto, según Hernández (2010).

Se agruparon los reportes recogidos producto de los grupos focales. Además, a partir de Bonilla (1997) se utilizó como recurso metodológico el análisis matricial propuesto, el cual permite evidenciar las categorías del estudio (Bonilla, 1997: 146). 
Floralba Barrero Rivera, Claudia Barrero Espinosa, Harold Borja Cely \& Merfi Raquel Montaño Sinisterra

\section{Procedimiento}

Inicialmente se hizo una revisión del estado del arte del tema en cuestión y se construyó el marco teórico. Posteriormente, se diseñó, elaboró y validó la encuesta, luego se realizó la transcripción de la información recolectada, desde los grupos focales $\mathrm{y}$, finalmente, se analizaron los resultados en dos momentos, así:

1) análisis de estadística descriptiva, y

2) Análisis matricial.

Los resultados luego se procesaron mediante el software SPSS, versión 18; y los grupos focales se examinaron desde la técnica de análisis de contenido.

\section{Resultados}

A continuación se presentan los resultados a partir de la encuesta respondida por 77 desertores quienes voluntariamente conformaron la muestra. Asimismo, la información lograda desde los grupos focales. Los resultados están dados en dos apartados; el primero contiene la información de deserción estudiantil, la deserción de acuerdo con factores de riesgo y el comportamiento de la deserción en el interior de los factores. El segundo apartado contiene las matrices de los grupos focales desde el análisis de las categorías orientadoras del estudio y con el procesamiento de la técnica de análisis de contenido.

\section{Información deserción estudiantil}

\begin{tabular}{|ccc|}
\hline Semestre & Frecuencia & Porcentaje \\
\hline 4 & 50 & 66.6 \\
\hline $5-8$ & 14 & 18.6 \\
\hline $8-10$ & 3 & 4.0 \\
\hline No aplica & 8 & 10.6 \\
\hline
\end{tabular}

Tabla 1. Semestre en que se efectuó el retiro de la universidad

De acuerdo con la tabla anterior se puede evidenciar que $66.6 \%$ de los estudiantes se retira en los primeros cuatro semestres de la carrera y sólo el $4 \%$ se retira entre octavo y décimo semestre.

\begin{tabular}{|c|c|c|}
\hline Respuesta & Frecuencia & Porcentaje \\
\hline Sí & 22 & 29,3 \\
\hline No & 53 & 70,6 \\
\hline
\end{tabular}

Tabla 2. Me retiré de la Universidad para ingresar a otra institución de Educación Superior

Según esta tabla, 29,3\% de los estudiantes que desertan en la universidad lo hacen para ingresar a otra IES, mientras que $70,6 \%$ se retira sin un proyecto de continuar estudiando

\begin{tabular}{|c|c|c|}
\hline Respuesta & Frecuencia & Porcentaje \\
\hline Sí & 6 & 8 \\
\hline No & 69 & 92 \\
\hline
\end{tabular}

Tabla 3. Deserción precoz. Alguna vez fui admitido a un programa de Educación Superior y nunca proseguí a matricularme

De acuerdo con la tabla anterior, se observa que sólo $8 \%$ de los desertores participantes en algún momento de su vida fueron admitidos a un programa de Educación Superior sin proceder a matricularse, el $92 \%$ restante niega este comportamiento.

\section{Deserción estudiantil de acuerdo con los factores de riesgo}

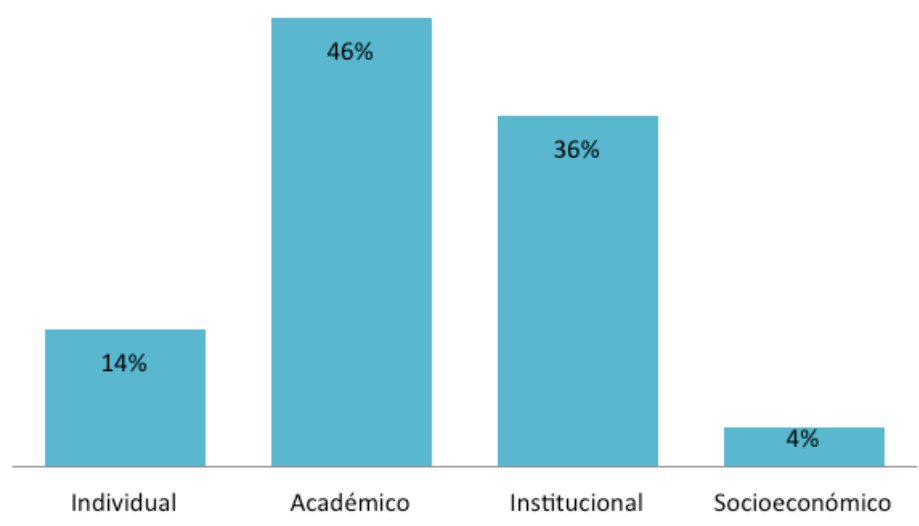

Figura 1. Factores de riesgo de deserción en estudiantes mujeres

En cuanto a las mujeres se puede afirmar que los factores con prevalencia a la hora de tomar la decisión de retirar de la carrera, son el académico (46\%) seguido del institucional (36\%); el factor de menor incidencia de acuerdo con las mujeres es el socioeconómico (4\%) seguido del individual (14\%). 


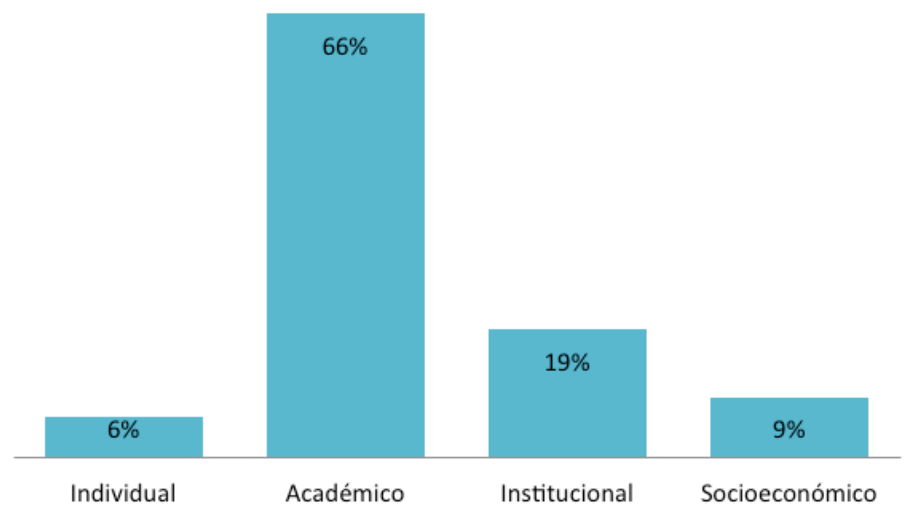

Figura 2. Factores de deserción en estudiantes hombres

En cuanto a los hombres, se evidencia una prevalencia de los factores académico (66\%) seguido del factor institucional (19\%); sin embargo, se muestra que el factor que menos influye en esta población es el personal (6\%) seguido del socioeconómico $(9 \%)$.

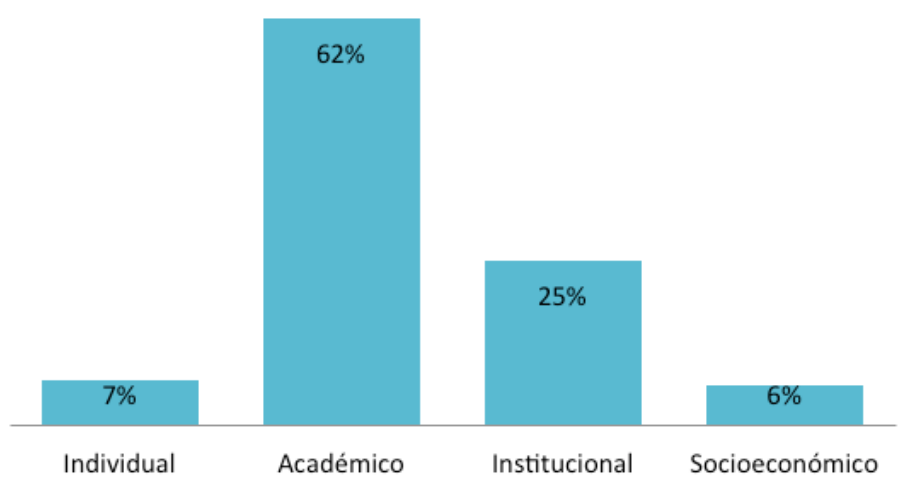

Figura 3. Factores de deserción en estudiantes de estrato socioeconómico bajo

En relación con los estudiantes pertenecientes a un estrato socioeconómico bajo, se puede observar que el factor con más influencia es el académico (62\%), además de ello, se evidencia que el factor con menos prevalencia es el socioeconómico $(6 \%)$.

Como se muestra en la figura 4, los factores académicos e institucionales son lo de mayor prevalencia en población de estrato socioeconómico medio, así mismo, el factor de menor prevalencia es el correspondiente al socioeconómico.

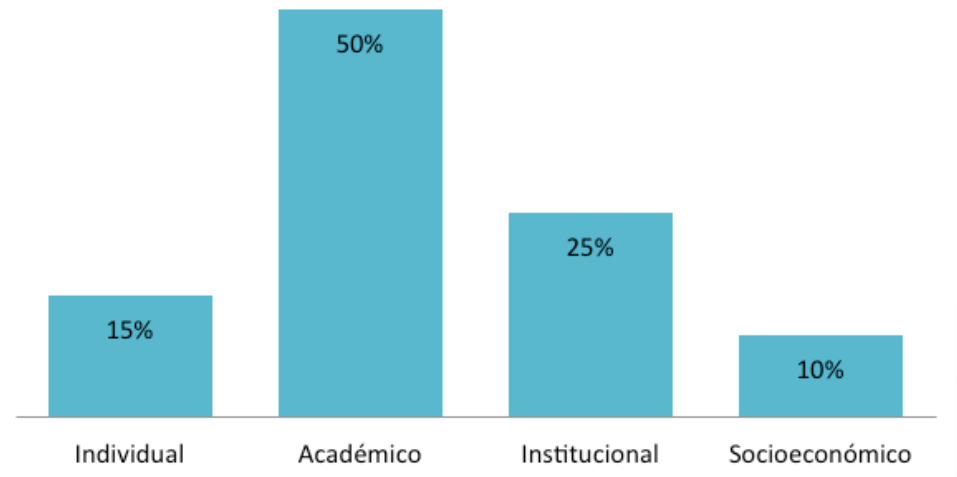

Figura 4. Factores de deserción en estudiantes de estrato socioeconómico medio.

\section{Comportamiento de la deserción en el interior de los factores}

\begin{tabular}{|c|c|c|}
\hline Ítem & N & Porcentaje de Sí \\
\hline 1 & 42 & 56 \\
\hline 2 & 27 & 36 \\
\hline 3 & 28 & 38 \\
\hline 4 & 9 & 12 \\
\hline 5 & 20 & 27 \\
\hline 6 & 15 & 20 \\
\hline 7 & 26 & 35 \\
\hline 8 & 22 & 29 \\
\hline 9 & 14 & 19 \\
\hline 10 & 20 & 27 \\
\hline 11 & 24 & 32 \\
\hline 12 & 28 & 38 \\
\hline 13 & 22 & 29 \\
\hline 14 & 38 & 51 \\
\hline 15 & 11 & 15 \\
\hline 16 & 21 & 28 \\
\hline 17 & 36 & 48 \\
\hline 18 & 13 & 17 \\
\hline
\end{tabular}

Tabla 4. Comportamiento de los ítemes, factor de riesgo individual

De acuerdo con esta tabla se encuentra que en el interior del factor individual, el ítem que más frecuencia presenta es el 2, es decir que los estudiantes desertan por factores emocionales (24\%) seguido de problemas de salud (16\%), dificultades de integración (11\%). La causa por la que menos abandonan los estudios se relaciona con el consumo de alcohol (1\%). 
Floralba Barrero Rivera, Claudia Barrero Espinosa, Harold Borja Cely \& Merfi Raquel Montaño Sinisterra

\begin{tabular}{|c|c|c|}
\hline Ítem & $\mathbf{N}$ & Porcentaje de Sí \\
\hline 1 & 12 & 16 \\
\hline 2 & 18 & 24 \\
\hline 3 & 9 & 11 \\
\hline 4 & 7 & 9 \\
\hline 5 & 6 & 8 \\
\hline 6 & 5 & 7 \\
\hline 7 & 6 & 8 \\
\hline 8 & 1 & 1 \\
\hline 9 & 4 & 5 \\
\hline
\end{tabular}

Tabla 5. Comportamiento de los ítemes, factor de riesgo académico

Según esta tabla, se encuentra que en el interior del factor académico y teniendo en cuenta los ítemes en sentido negativo, la causa por la que más desertan los estudiantes están relacionadas con el incumplimiento de las propias expectativas por parte del programa académico, la repitencia de años en el colegio, rendimiento regular en la carrera, como también iniciar diferentes programas.

\begin{tabular}{|c|c|c|}
\hline Ítem & N & Porcentaje de Sí \\
\hline 1 & 14 & 19 \\
\hline 2 & 16 & 21 \\
\hline 3 & 9 & 12 \\
\hline 4 & 22 & 29 \\
\hline 5 & 43 & 58 \\
\hline 6 & 38 & 51 \\
\hline 7 & 18 & 24 \\
\hline 8 & 22 & 29 \\
\hline 9 & 22 & 29 \\
\hline 10 & 26 & 35 \\
\hline 11 & 12 & 16 \\
\hline 12 & 16 & 21 \\
\hline 13 & 6 & 8 \\
\hline 14 & 9 & 12 \\
\hline 15 & 12 & 16 \\
\hline 16 & 10 & 10 \\
\hline 17 & 18 & 24 \\
\hline 18 & 22 & 29 \\
\hline & & \\
\hline
\end{tabular}

Tabla 6. Comportamiento de los ítemes, factor de riesgo institucional

De acuerdo con esta tabla se encuentra que en el interior del factor institucional y teniendo en cuenta los ítemes en sentido negativo, la causa por la que más desertan los estudiantes están relacionadas con las dificultades para llegar a la universidad, asimismo en la relación con profesores.

\begin{tabular}{|c|c|c|}
\hline Ítem & $\mathbf{N}$ & $\begin{array}{c}\text { Porcentaje } \\
\text { de Sí }\end{array}$ \\
\hline 1 & 44 & 59 \\
\hline 2 & 28 & 38 \\
\hline 3 & 15 & 20 \\
\hline 4 & 11 & 15 \\
\hline 5 & 37 & 50 \\
\hline 6 & 21 & 28 \\
\hline 7 & 18 & 24 \\
\hline 8 & 46 & 62 \\
\hline 9 & 42 & 56 \\
\hline 10 & 27 & 36 \\
\hline 11 & 10 & 13 \\
\hline 12 & 4 & 5 \\
\hline 13 & 23 & 32 \\
\hline 14 & 9 & 12 \\
\hline 15 & 5 & 7 \\
\hline
\end{tabular}

Tabla 7. Comportamiento de los ítemes, factor de riesgo económico

De acuerdo con esta tabla en el interior del factor de riesgo socioeconómico y teniendo en cuenta los ítemes negativos se encuentra que los estudiantes desertan por dificultades económicas de la familia, tienen padres que no son profesionales, el costo del transporte diario, vivir lejos de la universidad y quedarse sin trabajo.

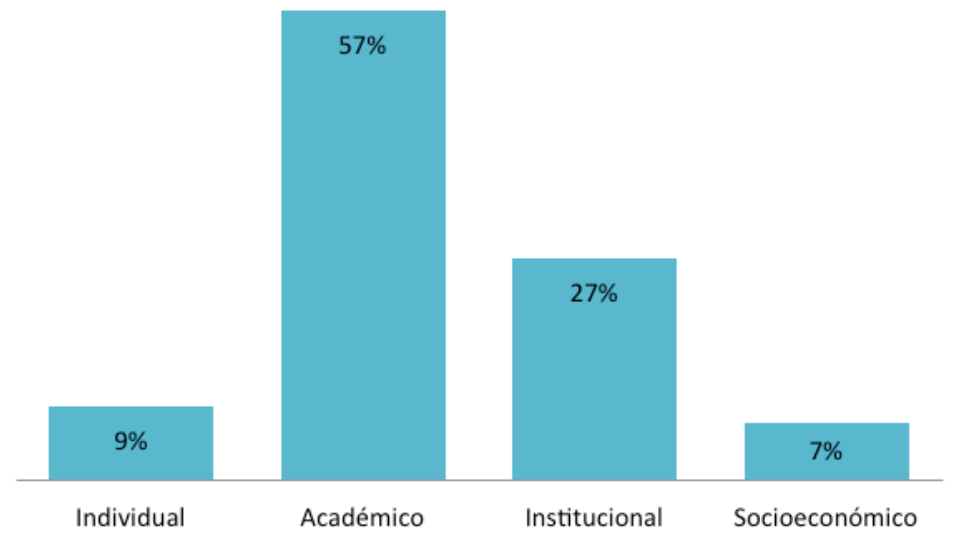

Figura 9. Resultado general, factores de deserción en estudiantes

Tal como se observa en la figura 5, para la población en general los factores con mayor prevalencia son el académico (57\%), seguido por el institucional (27\%). Los factores de menor prevalencia son el socioeconómico (7\%) y el individual (9\%). 


\section{Resultados cualitativos}

Los grupos focales se realizaron con directores de programa y con profesores que desempeñan el rol de acompañar a estudiantes. Los participantes del grupo focal fueron cinco facultades (Ingeniería, Psicología, Ciencias Empresariales, Ciencias Jurídicas y Educación). La información recogida se procesó desde el análisis de contenido. Según Hernández (2010) y Avela (2003), esta técnica se define como la posibilidad de acceder de manera sistemática a tres niveles (superficial, analítico e interpretativo) y de construir a partir de allí un texto distinto de los anteriores. La transcripción del grupo focal permite clasificar e ir reduciendo los datos de manera sistemática y ordenada.
Los resultados cuantitativos con los cualitativos permiten ser complementados dado que se encontraron unidades de análisis comunes entre las dos perspectivas del fenómeno. Situaciones académicas asociadas a la falta de orientación vocacional, a vacíos de conocimiento que traen de la secundaria, a dificultades de socialización por parte de los estudiantes. Todas estas situaciones asociadas a la familia y a la sociedad quedan reflejadas en las percepciones de los participantes a los distintos grupos focales. La deserción estudiantil es una problemática compleja que se deriva de la sumatoria de factores de origen variado. Sin embargo, esta investigación que desde la metodología tanto cuantitativa como cualitativa abordó el fenómeno, corrobora que en la Universidad de San Buenaventura, sede Bogotá, predominan las condiciones del estudiante

\begin{tabular}{|c|c|c|c|}
\hline Eje & Factor & Recurrencias & Teoría \\
\hline \multirow[t]{5}{*}{$\begin{array}{c}\text { Factores } \\
\text { de riesgo } \\
\text { asociados a } \\
\text { la deserción } \\
\text { estudiantil } \\
\text { universitaria }\end{array}$} & Individual & $\begin{array}{l}\text { Incoherencia entre las expectativas del estudiante y las } \\
\text { demandas reales de la carrera. } \\
\text { La falta de orientación vocacional. } \\
\text { (Facultades de Ingeniería, Educación, Psicología y } \\
\text { Ciencias Jurídicas). }\end{array}$ & $\begin{array}{l}\text { Falta de actitud y compromiso por parte de los } \\
\text { estudiantes, su inmadurez y falta de dedicación; el } \\
\text { bajo rendimiento académico, el desconocimiento de } \\
\text { los programas académicos y sus correspondientes } \\
\text { metodologías, la selección inadecuada de la carrera, } \\
\text { la falta de orientación vocacional, los vacíos en la } \\
\text { preparación secundaria. (Basualdo, 2005) }\end{array}$ \\
\hline & Socioeconómico & $\begin{array}{l}\text { Pocos recursos económicos y dificultades de orden social. } \\
\text { Situación laboral económica de las familias. } \\
\text { (Facultades de Ingeniería, Educación, Psicología y } \\
\text { Ciencias Jurídicas). }\end{array}$ & $\begin{array}{l}\text { El factor financiero aparece de manera recurrente en la } \\
\text { deserción estudiantil y se relaciona con la imposibilidad } \\
\text { de pagar los estudios, por lo cual a menudo el } \\
\text { estudiante se ve abocado a trabajar para su propio } \\
\text { sustento, hecho que a su vez incidió en el tiempo de } \\
\text { dedicación para su estudio(Fernández, 2008) }\end{array}$ \\
\hline & Académico & $\begin{array}{l}\text { Orientación vocacional } \\
\text { Dificultades en lectura y escritura. } \\
\text { Trabajo laboral y académico simultáneo } \\
\text { Vacío de conocimiento en la formación secundaria } \\
\text { (Facultades de Ingeniería, Ciencias Empresariales y } \\
\text { Educación) }\end{array}$ & $\begin{array}{l}\text { Vacíos de preparación secundaria asociados a los } \\
\text { problemas de matemáticas, lectura y escritura inciden } \\
\text { en la deserción estudiantil Asimismo, la metodología de } \\
\text { los profesores, las estrategias de enseñanza empleadas } \\
\text { por los docentes afectan este factor (Calderón, 2005) }\end{array}$ \\
\hline & Institucional & $\begin{array}{l}\text { Sentido de pertenencia. } \\
\text { Faltan espacios diferentes de los académicos que } \\
\text { permitan el complemento de su formación. Es el caso de } \\
\text { deportes, espacios culturales y esparcimiento, entre otros. } \\
\text { A los aspirantes de los diferentes programas de las } \\
\text { facultades no se les informa lo suficiente de la carrera } \\
\text { en la que están interesados (Facultades de Ingeniería, } \\
\text { Ciencias Empresariales y Ciencias Jurídicas). } \\
\text { Ubicación geográfica de la Universidad. } \\
\text { Inseguridad alrededor de la Universidad }\end{array}$ & $\begin{array}{l}\text { Factor vinculado con la adaptación del estudiante al } \\
\text { ambiente universitario. En esta categoría se tienen } \\
\text { en cuenta aspectos como la mortalidad académica, } \\
\text { las becas y las formas de financiamiento de la } \\
\text { Universidad, los recursos universitarios, la calidad de } \\
\text { los programas, el nivel de interacción personal con los } \\
\text { profesores y estudiantes. La naturaleza de la institución } \\
\text { y sus proyecto educativo, misión y visión (Centro } \\
\text { de Estudios sobre Desarrollo Económico-CEDE), } \\
\text { Universidad de los Andes (2007) }\end{array}$ \\
\hline & Conclusiones & $\begin{array}{l}\text { Se encontró una unidad de análisis común en todas } \\
\text { las facultades y se referencia en cuanto a orientación } \\
\text { vocacional y } \\
\text { desconocimiento de los estudiantes de los servicios de la } \\
\text { Universidad. }\end{array}$ & $\begin{array}{l}\text { La deserción estudiantil es compleja y multicausal. } \\
\text { (Tinto, 2002, 2006, 2010) }\end{array}$ \\
\hline
\end{tabular}

Matriz 1. Consolidado información general grupos focales

Fuente: Elaboración propia (2014). 
con la preparación académica previa en donde se evidencian dificultades en torno a las competencia de lectura y escritura. Por otra parte, las condiciones de la institución, y sus propias características reflejan que hay estudiantes que no conocen los beneficios que les brinda la institución.

\section{Discusión}

La presente investigación tuvo como objetivo identificar los factores de riesgo asociados a la deserción estudiantil universitaria en los programas de pregrado de la Universidad de San Buenaventura, sede Bogotá, con el fin de detectar la situación real y brindar aportes desde la psicología educativa, de tal modo que contribuya a la comprensión del fenómeno. Este apartado presenta las reflexiones que privilegia conocer dichos factores desde el contexto propio, por cuanto la tradición investigativa del tema actualmente destaca los programas de retención como manera de abordar el problema de la deserción. No obstante, esta investigación presenta una forma de comprender el fenómeno a partir de la identificación y la intervención de cada uno de los factores desde los modelos de la psicología educativa.

En el caso de la presente indagación se entrega un instrumento validado por el grupo investigador que contribuye al análisis de la deserción de manera contextual, periódica y sistemática. Los ítemes del instrumento fueron diseñados por profesores investigadores con experiencia en acompañamiento a estudiantes. Al formular las preguntas, éstas fueron inspiradas en quejas propias y sentimientos generados por el riesgo de la deserción estudiantil en nuestros estudiantes bonaventurianos. Si bien es cierto, es necesario afirmar que permiten estos ítemes cotejar la investigación en el tema, tal como lo afirman Tinto (2002), y Cabrera et al (2000), “[...] los factores de riesgo son individuales, institucionales, académicos y socioeconómicos" (Tinto, 2002: 56).

En ese orden, la presente investigación deja como recomendación rastrear periódicamente a los estudiantes en las diferentes cohortes e identificar las razones de por qué se van (aplicando la encuesta). Una vez conocidos los resultados se convierte en camino para sistematizar el fenómeno e intervenirlo de manera oportuna y propositiva. Por otra parte, el instrumento permite identificar el comportamiento del tipo de desertor durante el ciclo académico donde es más frecuente su retiro y como lo denominan los investigadores, deserción precoz, temprana, tardía. Estudios como los de Ramírez (2002) afirman que es relevante incluir el papel de la formación impartida por las instituciones educativas de nivel básico medio y su incidencia en los vacíos de conocimiento que presentan los estudiantes al ingresar a la universidad.

Por otra parte, afirma el investigador conocer y abordar las prácticas pedagógicas de los docentes son claves para ayudar a prevenir la deserción. Asimismo, Donoso y Schiefelbein (2007) añaden que los niveles de déficit asociados a las condiciones de entrada de los estudiantes tienen que examinarse desde su cultura y la cultura organizacional de la institución.

Estas investigaciones conducen a conocer los tipos de desertores en los distintos momentos del ciclo académico, permitiendo no caer en generalizaciones que hacen daño a la realidad del fenómeno. Existen muchos tipos de motivos por los cuales se retiran los estudiantes; éstos van desde el motivo forzoso hasta el voluntario. En la misma universidad pueden cambiarse de programas sin que por eso se consideren desertores.

Algunas instituciones educativas se refieren a los estudiantes generalizando la deserción sin conocer exactamente la clasificación del fenómeno. Por tanto, ubicar el problema de la deserción desde la propia realidad permite organizar y conocer el comportamiento del tema y generar programas articulados a modelos probados que favorezcan la permanencia de los estudiantes.

Los modelos como los de Spady (1970), Ethington (1990) y Pascarella \& Terenzine (1991), entre otros, permiten proponer una salida para intervenir cada uno de estos factores. Dichos modelos ofrecen una ruta para abordar cada factor de riesgo. Si se logra aplicarlo y sistematizarlo periódicamente se estaría trabajando en dos aspectos: 


\section{Conocer y ordenar el fenómeno}

2. Intervenirlo de manera oportuna desde los avances de la investigación en el tema y no desde programas que correspondan a la coyuntura de la deserción.

Estos aspectos podrían contribuir a sistematizar las experiencias favoreciendo planes de mejoramiento continuo y permitiendo a largo plazo la elaboración y validación de un modelo propio para abordar la deserción estudiantil para la Universidad de San Buenaventura, sede Bogotá.

Es conveniente asimismo afirmar que los resultados de esta investigación son producto de una muestra no significativa por cuanto se encontraron dificultades en la consecución de las bases de datos y la muestra de desertores. Por tanto, no se puede concluir que los hallazgos son tendencias definitivas en los factores de riesgo con respecto a la institución en el período comprendido entre 2009 y 2013. Desde este argumento estos resultados no se pueden generalizar, pero sí se pueden analizar. En este sentido, se pueden precisar las siguientes reflexiones:

1. Las bases de datos de los estudiantes desertores deben estar articuladas desde la unidad de registro y control con el Centro de Bienestar Universitario. Esto permitiría contar con una base completa que facilite el contacto permanente con los estudiantes y que a su vez permita la actualización frecuente de la misma.

2. Con el fin de contribuir a no romper el vínculo de los estudiantes con la institución de manera definitiva, sería importante diseñar programas culturales y sociales que permitan la relación de ellos con la institución. La experiencia de contactar a estudiantes desertores a pesar de contar con las bases de datos se torna compleja, pues no responden a los correos y mucho menos a las llamadas personales.

3. Generar programas de participación para toda la población estudiantil incluyendo los desertores ofrecería una mirada diferente de este estudiante retirado. "Es dejar siempre abierta la posibilidad de un regreso".

4. En este marco de reflexiones se puede afirmar que los 100 estudiantes del estudio que diligenciaron la encuesta y una vez realizada la depuración de la base de datos, sólo se contó con 77 estudiantes. Esto corrobora lo expuesto anteriormente: el estudiante queda sin ningún vínculo social y afectivo con la Universidad. Se entiende que éste como estudiante de un programa se pierde, pero debería permanecer algún motivo para no abandonar totalmente a la institución.

5. No fue posible identificar el comportamiento de los desertores en el rango comprendido entre 2009 y 2013, por cuanto la muestra que finalmente se obtuvo precisó que muchos de los participantes omitieron esta información, razón por la cual se hace imposible conocer los datos acerca de este aspecto de la investigación.

Finalmente, los resultados mostraron una tendencia no definitiva, pero sí susceptible al análisis como es el factor de riesgo académico, el cual permite identificar serios vacíos de conocimiento en la formación media y dificultades también de orden vocacional. Las prácticas pedagógicas de los docentes son condiciones asociadas a este factor.

Por otra parte, en el factor de riesgo institucional que puntuó como segundo deja ver la necesidad de promover mejores espacios extracurriculares que favorezcan la identidad de los estudiantes con la institución. También es necesario ofrecer posibilidades de financiación más contundentes, acompañadas por un plan de subsidios. Estos aspectos mencionados fueron corroborados por los grupos focales donde se recomendaron puntos concretos para mejorar el factor de riesgo académico e institucional. A continuación se menciona un apartado concreto:

[... "Un primer y claro aspecto es el tema de orientación vocacional que se encuentra en el estudiante que no trae unos claros y sólidos intereses profesio- 
nales, manifestado en "el muchacho que viene a la universidad porque sí y viene a estudiar psicología porque sí”. El tema vocacional está relacionado con muchas otras cosas como por ejemplo: el desconocimiento de la universidad, de la carrera y del rol profesional, lo que lleva a tener una visión sesgada de la carrera limitando los intereses profesionales.

En menor porcentaje que el anterior un segundo aspecto viene a ser el "potencial académico del estudiante", esto sucede cuando llegan estudiantes con dificultades de lectura y escritura y se encuentran con que en la carrera deben realizar sintesis y argumentaciones por ejemplo, lo que sobrepasa sus capacidades entonces deciden renunciar (fragmento del grupo focal, Facultad de Psicología, septiembre 11 de 2014).

Por otra parte, el factor de riesgo institucional obtuvo importantes percepciones en el grupo focal. A continuación se extrae el siguiente fragmento:

[...] En la inducción a estudiantes nuevos no hay sensibilización a los estudiantes en lo que va a ser la carrera. Cuando la Universidad ha hecho inmersión en diferentes programas de las facultades (del colegio a la universidad) no es muy claro el seguimiento que se hace. Los profesores de los cursos que ellos realizan hacen la observación de que son juiciosos y aplicados; pero en la mayoría de los casos estos estudiantes no se retienen y se van. Otro factor que ha sido notorio es que los estudiantes no tienen sentido de pertenencia. A algunos no les gustan las materias de humanidades. Faltan espacios diferentes a los académicos que permitan el complemento de su formación. Es el caso de deportes, espacios culturales, de esparcimiento entre otros (fragmento del grupo focal realizado a las Ingenierías, 11 de septiembre de 2014).

Estos resultados validan la literatura actual acerca del tema, tal como lo afirman las investigaciones de Tinto (2002), Cabrera (2004) y Donoso (2007) del Centro de Desarrollo, Universidad de los Andes, 2007). Todos aseveran que la deserción es compleja como fenómeno pero que el factor de riesgo más contundente tiene que ver con el académico. Sin embargo, es necesario tener en cuenta que todos los factores unidos propician el detonante de la deserción estudiantil.

Por consiguiente, se deja abierta la posibilidad para continuar trabajando futuras investigaciones en el tema que lleven a complementar esta iniciativa que no es la primera en la institución, pero que puede contribuir a comprender el fenómeno de la deserción desde el presente abordaje del estudio.

Esta configuración convertiría la debilidad del factor en oportunidad de intervenir con programas educativos asociados a modelos, mas no a situaciones coyunturales que redundan en resultados a corto plazo al igual que a abordar con seriedad y profundidad resultados estructurales de una problemática bastante compleja.

\section{Referencias}

Avela, J. (2003). Las técnicas de análisis de contenido: una revisión actualizada. En http// www.fundcion. org/PDF, recuperado el 16 de Agosto del 2014.

Basualdo, M. (2005). Deserción y repotencia en la Educación Superior universitaria en Paraguay. Observatorio de Educación Superior en América Latina y el Caribe.

Berger, J. and Millem (2000). Organizacional behavior in Higher Education and student outcomes. In: J. Smart (Ed), Higher Education: Handbook of theory and research. Vol. 15: 268-338.

Bonilla, E. (1997). Más allá del dilema de los métodos. México:

Cabrera (1992). The role of finances on the persistence process. A structural model. Research in Higher Education. Vol. 33, N 5: 303- 336.

Cabrera et al (2000). Economic influences on persistence. En J. M. Braxton. Reworking the student 


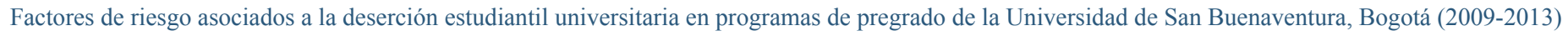

departure puzzle: New theory and research on college student retention (p.20-47). Nashville: Vanderbilt University Press.

Calderón, J. (2005). Estudio sobre la repitencia y deserción en la Educación Superior de Guatemala. Observatorio de la Educación Superior en América Latina y el Caribe. IESALC/Unesco. Recuperado el 15 de Octubre del 2014, en http// www.nd.edu/-cmendoz1/educaciónsuperior.pdf.

Centro de Estudios sobre Desarrollo Económico, Universidad de los Andes (2007). Investigación sobre deserción en las instituciones de Educación Superior en Colombia. Bogotá: Cede, Uniandes.

Colombia Ministerio de Educación Nacional (2004) Resolución por la cual se ordena la apertura del Concurso de Méritos No CPM-MEN-01-04. Recuperado el 20 de agosto de 2014 de htpp/ menweb.muneducación.gov.co/contratos/pdfs/ resolución_aperturacp.doc

Colombia Ministerio de Educación Nacional (2007). Proyecto estrategias para disminuir la deserción en la Educación Superior. Bogotá: MEN.

Donoso, S \& Schiefelbein, E. (2007). Análisis de los modelos explicativos de retención de estudiantes en la Universidad: una visión desde la desigualdad social. Estudios pedagógicos. Recuperado el 12 de Septiembre del 2014, de http: www.cielo.

Ethington, C. (1990). A psychological model of student persistence. Research in Higher Education, 3 (31), $279-293$.

Fishbein \& Ajzen (1975). Attitudes toward objects as predictors of simple and multiple behavioral criteria. Psychological Review. N 81: 59-74.

Gómez, H (1998). Educación: la agenda del siglo XXI: hacia un desarrollo humano. Bogotá: tercer mundo editores.

Hernández R (2010) Metodología de la Investigación cientifica. México: McGraw Hill.

Instituto colombiano para el fomento de la Educación Superior (2003). La Educación Superior en Colombia Década de los 90. Bogotá: ICFES.

Ramírez, L. (2002). Reflexiones sobre la deserción y la mortalidad estudiantil en las universidades colombianas: un debate necesario. Revista Educación y Educadores, 5, 21-38.

Redondo, J M(1999). La dinámica escolar: de la diferencia a la desigualdad. Revista de Psicología de la Universidad de Chile. Vol. 6.

Pascarella, E, \& Terenzini, P. (1991). How college affects students: findings and insights from twenty years of research. San Francisco: Jossey- Bass.

Sandoval, C (2002). La investigación cualitativa. Bogotá: Icfes.

Spady, W. (1970). Dropouts from higher education: An interdisciplinary review and synthesis. Interchange. Vol. 19, N 1 1: 109-121.

Tinto, V. (1993). Leaving College: Rethinking the Causes and Cures of Student Attrition. Chicago: The University of Chicago Press.

Tinto, V. \& Giovagnoli (2002). Epilogue: Moving from Theory to Action. En A. Seidman (Ed.). College Student Retention: Formula for Student Success (pp. 317-334). Westport, CT: ACE/Praeger. 